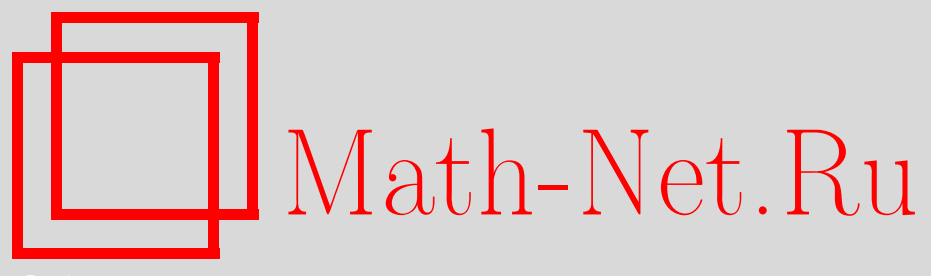

В. В. Беняш-Кривец, В. П. Платонов, $S$-единицы в гиперэллиптических полях, УМН, 2007, том 62, выпуск 4, 149-150

DOI: https://doi.org/10.4213/rm7260

Использование Общероссийского математического портала Math-Net.Ru подразумевает, что вы прочитали и согласны с пользовательским соглашением http://www . mathnet.ru/rus/agreement

Параметры загрузки:

IP : 3.91 .87 .62

26 апреля 2023 г., 03:15:23

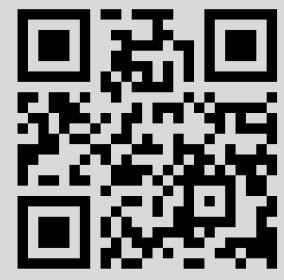




\section{$S$-единицы в гиперэллиптических полях}

\section{В. В. Беняш-Кривец, В. П. Платонов}

В настоящей заметке мы представляем некоторые результаты о вычислении групп $S$-единиц в гиперэллиптических полях.

Пусть $k=\mathbb{F}_{q}(x)$ - поле рациональных функций от одной переменной над конечным полем $\mathbb{F}_{q}$ характеристики $p>2$ и $d(x)=a_{0} x^{2 n+1}+a_{1} x^{2 n}+\cdots+a_{2 n+1} \in \mathbb{F}_{q}[x]$ - свободный от квадратов многочлен, $a_{0} \neq 0$. Пусть $K=k(\sqrt{d})$. Для неприводимого многочлена $v \in \mathbb{F}_{q}[x]$ через $|\cdot|_{v}$ будем обозначать соответствующее нормирование на $k$. Пусть $(\alpha, \beta)$ - точка на кривой $y^{2}=d(x), \beta \neq 0$. Тогда нормирование $|\cdot|_{x-\alpha}$ имеет два продолжения на $K$. Эти продолжения будем обозначать $|\cdot|_{1}$ и $|\cdot|_{2}$. Неархимедово нормирование $|\cdot|_{\infty}$ имеет единственное продолжение на $K$, которое по прежнему будем обозначать через $|\cdot|_{\infty}$. Следующие два случая являются базисными для исследования $S$-единиц: 1) $S=\left\{|\cdot|_{\infty},|\cdot|_{1}\right\}$; 2) $S=\left\{|\cdot|_{\infty},|\cdot|_{1},|\cdot|_{2}\right\}$. Пусть $\mathscr{O}_{S}-$ кольцо $S$-целых элементов в $K$, т. е. таких элементов $y \in K$, что $|y|_{v} \geqslant 0$ для всех нормирований $|\cdot|_{v}$ поля $K$, не принадлежащих $S$. Множество обратимых элементов $U_{S}$ кольца $\mathscr{O}_{S}$ называется группой $S$-единиц поля $K$. В силу обобщенной теоремы Дирихле о единицах (см. [1; гл. IV, теорема 9]) группа $U_{S}$ является прямым произведением группы $\mathbb{F}_{q}^{*}$ и свободной абелевой группы $G$ ранга $|S|-1$. Независимые образующие группы $G$ называются фундаментальными $S$-единицами.

В классическом случае квадратичного расширения $L=\mathbb{Q}(\sqrt{d})$ поля $\mathbb{Q}$ фундаментальную единицу поля $L$ можно найти, используя разложение $\sqrt{d}$ в цепную дробь [2]. Однако для функциональных полей метод цепных дробей не всегда позволяет найти фундаментальную единицу. Цель настоящей заметки - найти алгоритм для вычисления фундаментальных $S$-единиц гиперэллиптического поля $K$ в указанных двух случаях.

Первое предложение носит технический характер.

Предложение 1. Пусть $y=f+g \sqrt{d}$, где $f, g \in \mathbb{F}_{q}[x], f \neq 0, g \neq 0,(f, g)=1$, и пусть $v \in \mathbb{F}_{q}[x]$ - неприводимый многочлен. Тогда справедливы следующие утверждения.

1. Если $|\cdot|_{v}$ имеет два продолжения $|\cdot|_{v^{\prime}} u|\cdot|_{v^{\prime \prime}}$ на $K$, то либо $|y|_{v^{\prime}}=0$, либо $|y|_{v^{\prime \prime}}=0$.

2. Если $v \nmid d u|\cdot|_{v}$ имеет единственное продолжение $|\cdot|_{v^{\prime}}$ на $K$, mо $|y|_{v^{\prime}}=0$.

3. Если $v \mid d u v \nmid f$, то $|\cdot|_{v}$ имеет единственное продолжение $|\cdot|_{v^{\prime}}$ на $K u|y|_{v^{\prime}}=0$.

4. Если $v|d u v| f$, то $|\cdot|_{v}$ имеет единственное продолжение $|\cdot|_{v^{\prime}}$ на $K u$ $|y|_{v^{\prime}}=1 / 2$.

Следующее предложение характеризует $S$-целые элементы в $K$.

ПредЛОЖениЕ 2. Любой элемент $y \in \mathscr{O}_{S}$ имеет вид

$$
y=(f+g \sqrt{d})(x-\alpha)^{-m},
$$

где $f, g \in \mathbb{F}_{q}[x], m \geqslant 0$. Если при этом $m>0$ и $x-\alpha$ не делит одновременно $f$ и $g$, mo $x-\alpha \nmid f, x-\alpha \nmid g$.

Для доказательства предложения 2 достаточно заметить, что если знаменатель $y$ делится на неприводимый многочлен $v \neq x-\alpha$, то для некоторого продолжения $|\cdot|_{v^{\prime}}$ нормирования $|\cdot|$ в в силу предложения $1|y|_{v^{\prime}}<0$.

Отметим, что не любой элемент вида $(f+g \sqrt{d})(x-\alpha)^{m}$ является $S$-целым. Для дальнейшего нам важно знать, какие значения может принимать норменное отображение на $S$-единицах.

ПредлОЖениЕ 3. Если $\varepsilon=(f+g \sqrt{d}) /(x-\alpha)^{m_{1}} \in U_{S}$, где $f, g \in \mathbb{F}_{q}[x]$, u если $\varepsilon \notin \mathbb{F}_{q}^{*}$, mo $f \neq 0, g \neq 0,(f, g)=1 u N_{K / k}(\varepsilon)=a(x-\alpha)^{m}$, где $a \in \mathbb{F}_{q}^{*} u 0 \neq m \in \mathbb{Z}$. 
Так же, как и в случае $S$-целых элементов, если элемент $\varepsilon \in K$ обладает свойством $N_{K / k}(\varepsilon)=a(x-\alpha)^{m}$, то из этого не следует, что $\varepsilon$ является $S$-единицей. Однако следующее предложение показывает, что если норменное уравнение

$$
f^{2}-g^{2} d=a(x-\alpha)^{m},
$$

где $m \neq 0$ фиксировано, имеет решение в многочленах $f, g \in \mathbb{F}_{q}[x]$, то либо $f+$ $g \sqrt{d} \in U_{S}$, либо $f-g \sqrt{d} \in U_{S}$.

Предложение 4. Пусть $y=f+g \sqrt{d}$, где $f, g \in \mathbb{F}_{q}[x]$, u nусть $N_{K / k}(y)=$ $a(x-\alpha)^{m}$, где $a \in \mathbb{F}_{q}^{*} u m>0$. Тогда если $|y|_{2}=0$, mо $y=f+g \sqrt{d} \in U_{S}$, а если $|y|_{2}>0, m o(f+g \sqrt{d}) /(x-\alpha)^{m} \in U_{S}$.

Теорема 1. Пусть $S^{\prime}=\left\{|\cdot|_{\infty},|\cdot|_{1},|\cdot|_{2}\right\}, S=\left\{|\cdot|_{\infty},|\cdot|_{1}\right\}$. Тогда если $\varepsilon-\oint у н-$ даментальная $S$-единиц,

Для доказательства теоремы достаточно заметить, что если $\delta$ - произвольная $S^{\prime}$-единица, то, как и в случае $S$-единиц, $N_{K / k}(\delta)=a(x-\alpha)^{m}$. Тогда в силу предложения 4 либо $\delta \in U_{S}$, либо $\delta /(x-\alpha)^{m} \in U_{S}$. В первом случае $\delta=b \varepsilon^{t}$, а во втором случае $\delta=b \varepsilon^{t}(x-\alpha)^{m}$, где $b \in \mathbb{F}_{q}^{*}, t \in \mathbb{Z}$. Это и означает, что $\varepsilon, x-\alpha$ - независимые фундаментальные $S^{\prime}$-единицы.

Таким образом, в первую очередь нам необходимо уметь вычислить фундаментальную $S$-единицу. Для этого нужно найти минимальное натуральное $m$ такое, что уравнение (1) имеет решение в многочленах $f, g \in \mathbb{F}_{q}[x]$. Тогда либо $f+g \sqrt{d}$, либо $f-g \sqrt{d}$ является фундаментальной $S$-единицей.

Обозначим $v=x-\alpha$, и пусть $k_{v}$ - пополнение $k$ относительно нормирования $|\cdot|_{v}$. Поле $k_{v}$ можно отождествить с полем формальных степенных рядов $\mathbb{F}_{q}((v))$. Так как $|\cdot|_{v}$ имеет два продолжения на $K$, то $\sqrt{d} \in \mathbb{F}_{q}((v))$. Пусть $\sqrt{d}=\sum_{i=0}^{\infty} d_{i} v^{i}$. Следующая теорема дает алгоритм для нахождения фундаментальной $S$-единицы.

Теорема 2. Пусть $n=(\operatorname{deg} d-1) / 2$. Для челого числа $r \geqslant n$ определим матриць

$$
D_{r}=\left(\begin{array}{cccc}
d_{n+1} & d_{n+2} & \ldots & d_{r+1} \\
d_{n+2} & d_{n+3} & \ldots & d_{r+2} \\
\ldots & \ldots & \ldots & \ldots \\
d_{n+r} & d_{n+r+1} & \ldots & d_{2 r}
\end{array}\right), \quad H_{r}=\left(\begin{array}{cccc}
d_{n+2} & d_{n+3} & \ldots & d_{r+2} \\
d_{n+3} & d_{n+4} & \ldots & d_{r+3} \\
\ldots & \ldots & \ldots & \ldots \\
d_{n+r+1} & d_{n+r+2} & \ldots & d_{2 r+1}
\end{array}\right) .
$$

Норменное уравнение (1) с нечетным $m=2 r+1$ имеет решение в ненулевых многочленах $f, g \in \mathbb{F}_{q}[x]$ тогда и только тогда, когда $\operatorname{rank} D_{r}<r-n+1$, а с четным $m=2 r-$ тогда и толъко тогда, когда $\operatorname{rank} H_{r-1}<r-n$.

Если $K$ - поле эллиптической кривой, то матрицы $D_{r}, H_{r}$ являются квадратными. В этом случае определители специального вида, возникающие в теореме 2 , были впервые введены Якоби в XIX в. и носят название "персимметрический определитель" (persymmetric determinants), а матрицы такого вида называются “ганкелевыми матрицами" (Hankel matrices).

ЗАмечАниЕ. Мы ограничились здесь нормированиями, связанными с точками на гиперэллиптической кривой над полем $\mathbb{F}_{q}$. В действительности аналогичные результаты справедливы для любых нормирований поля $\mathbb{F}_{q}(x)$.

\section{Список литературы}

[1] А. Вейль, Основы теории чисел, Мир, М., 1972. [2] З. И. Боревич, И. Р. Шафаревич, Теория чисел, Наука, М., 1964.

В. В. Беняш-Кривец (V. V. Benyash-Krivets)

Белорусский государственный университет

E-mail: benyash@bsu.by

\section{В. П. Платонов (V.P. Platonov)}

Научно-исследовательский институт

системных исследований РАН

E-mail: platonov@niisi.ras.ru
Представлено В. М. Бухштабером Принято редколлегией 16.07.2007 